\title{
Criminologie
}

\section{Presse et délinquance ou comment lire entre les signes}

\section{Francine Soubiran-Paillet}

Volume 20, numéro 1, 1987

Faire les nouvelles : journalisme et affaires criminelles

URI : https://id.erudit.org/iderudit/017246ar

DOI : https://doi.org/10.7202/017246ar

Aller au sommaire du numéro

\section{Éditeur(s)}

Les Presses de l'Université de Montréal

ISSN

0316-0041 (imprimé)

1492-1367 (numérique)

Découvrir la revue

\section{Citer cet article}

Soubiran-Paillet, F. (1987). Presse et délinquance ou comment lire entre les signes. Criminologie, 20(1), 59-77. https://doi.org/10.7202/017246ar

\section{Résumé de l'article}

Based on two newspapers published in different socio-political contexts, one in Nice, France, the other in Geneva, Switzerland, we would like to compare the way these two dailies view crimes against property. Does the journalist report theft, breach of trust or break and enter in the same way? Are the same variables used in the articles or are important changes made from one article to the other? If such is the case, who orders the changes in the structure of the articles?

The work, which comprises a systematic list of six months daily articles, covers all typical situations published in the two newspapers. It seems, then, that the persons mentioned in the papers who belong to minority, as opposed to the majority groups, are generally presented as responsible for crime. It seems, too, that individuality disappears in the reconstruction of the reality by the media. All in all, the analysis shows that the press exercises only a relative influence on its readers. 


\section{PRESSE ET DÉLINQUANCE OU COMMENT LIRE ENTRE LES SIGNES Francine Soubiran-Paillet*}

Based on two newspapers published in different socio-political contexts, one in Nice, France, the other in Geneva, Switzerland, we would like to compare the way these two dailies view crimes against property. Does the journalist report theft, breach of trust or break and enter in the same way? Are the same variables used in the articles or are important changes made from one article to the other? If such is the case, who orders the changes in the structure of the articles?

The work, which comprises a systematic list of six months daily articles, covers all typical situations published in the two newspapers. It seems, then, that the persons mentioned in the papers who belong to minority, as opposed to the majority groups, are generally presented as responsible for crime. It seems, too, that individuality disappears in the reconstruction of the reality by the media. All in all, the analysis shows that the press exercises only a relative influence on its readers.

\section{INTRODUCTION}

Le discours médiatique, en particulier celui d'une presse régionale tenu à propos de l'atteinte aux biens est un discours relativement pauvre et récurrent. Il s'organise essentiellement au travers de l'exclusion d'un certain nombre de "coupables" désignés à la réprobation du lecteur, revêtus de signes. Ce discours est propice à la mise à nue des stratégies d'argumentation du journaliste mettant en scène des auteurs délinquants.

Telles furent nos constatations premières en étudiant deux journaux, l'un français à Nice (Nice-Matin), l'autre suisse à Genève, la Suisse. Deux quotidiens diffusés dans des climats socio-politiques contrastés.

Nous désirions savoir dans une optique comparative de quelle manière ces deux quotidiens appréhendaient le délinquant en nous cantonnant au domaine de l'atteinte aux biens. La question posée était au fond la suivante: à propos d'un vol, d'un abus de confiance ou d'un cambriolage, le journaliste parle-t-il toujours le même langage? Autrement dit: $y$-a-t-il un agencement de variables uniformes au sein des récits de presse ou rencontre-t-on au contraire des modifications d'un récit à l'autre? Qu'est-ce qui commande les modifications dans la structure des récits? Pour répondre, ici, nous avons pris en considération trois types d'infractions regroupées en trois rubriques:

*Francine Soubirau-Paillet est chercheur au Centre de recherches sociologiques sur le droit et les institutions pénales, à Paris. 
- la soustraction d'un bien à autrui sans violence: vols simples, vols qualifiés sans menace, ou atteinte à l'intégrité de la personne physique.

- les actes de brigandage, c'est-à-dire des actes de soustraction du bien d'autrui (hold-up en particulier) commis avec violence et/ou à main armée.

- les comportements relevant de la criminalité d'affaires.

Le corpus, qui porte sur un relevé systématique de six mois d'articles quotidiens, recouvre toutes les situations types évoquées par les deux journaux dans un contexte d'atteinte aux biens.

Nous montrerons comment les individus mis en scène dans les journaux et appartenant à des groupes minoritaires, par opposition à la majorité, elle, innocente, sont posés en responsables de la délinquance; comment l'individualité disparaît derrière le signe qui s'inscrit en creux dans le discours? (II) de quelle façon les médias reconstruisent le réel et quelles sont les règles auxquelles est soumise la mise en scène du crime? (III) étant entendu que le discours de presse comporte sa propre logique. Nous verrons enfin que ce discours de presse n'exerce pourtant qu'une influence relative sur ses lecteurs, allant ainsi dans le sens de travaux antérieurs (IV).

\section{RÉCIT DE PRESSE: USAGE ET RENFORCEMENT DES SIGNES D'EXCLUSION}

Nombreux sont les travaux européens menés à propos de la représentation du crime en général dans les médias. Nous en donnerons un bref aperçu.

En République fédérale d'Allemagne et en Autriche par exemple, les recherches effectuées démontrent d'abord: une déformation de la réalité quotidienne par les médias: les médias créent leur propre réalité.

- transmettent des éléments normatifs, des idéaux collectifs s'imposant au sein de la société. Parmi ces recherches, retenons celle de Stein-Hilbers (1977), concernant la télévision et se voulant systématique sur une période donnée;

- ce que donne à voir la télévision n'a pas grand chose de commun avec la réalité quotidienne des téléspectateurs;

- la télévision limite la présentation de la criminalité à des aspects partiels et spéciaux; 
- ça n'est qu'à des heures tardives que l'on diffuse des émissions qui, dans une certaine mesure, contiennent une information plus réaliste et plus nuancée sur la criminalité.

Sur les conditions générales journalistiques liées à la représentation du crime dans la presse, une étude de l'Institut de sociologie criminelle Ludwig Boltzmann à Vienne (1976) permet de faire le lien avec des travaux anglo-saxons puisqu'elle va dans le même sens que l'étude anglaise de Galtung et Ruge (1974): la priorité est accordée par le journaliste à des affaires criminelles non typiques.

Les travaux de Van Dijk (1980) aux Pays-Bas, conduits à partir de deux journaux très contrastés quant à leur contenu permettent de voir que l'un des deux journaux, le principal quotidien néerlandais, très populaire, donne surtout des informations de nature policière et un pourcentage moindre d'informations sur les procès criminels. $27 \%$ d'articles traitent du meurtre et de l'homicide. Les délits graves sont donc surreprésentés, alors que les délits d'affaires sont sous-représentés. Tous les articles traitent d'incidents et non de phénomènes généraux.

D’une façon générale, d'après Van Dijk, ce sont surtout des journaux populaires qui consacrent une grande partie de leur espace rédactionnel à la diffusion d'informations sur les crimes contre la vie.

Un recensement récent des travaux français effectués à propos du crime et des médias est fait par Robert (1984). Les travaux français dans ce champ concernent avant tout la presse écrite. Sans doute, dit Robert, parce que la démarche généralement adoptée est une démarche globalement inspirée de l'analyse de discours qui s'accommode mieux d'un corpus écrit. Lascoumes et Moreau-Capdevielle étudient la relation par la presse du procès Buffet-Bontemps (1976) s'appuyant sur l'analyse structurale du discours; Mouillaud (1981), l'évolution des attitudes et des langages de la presse écrite à l'égard de la limitation des naissances et de l'avortement durant la dernière décennie, également le rôle de la presse dans l'évolution des mours; Auclair (1982), s'attache à la relation journalistique d'un fait divers à épisodes.

Notre propre recherche est proche des travaux français précités, car il s'agit là encore d'une analyse de discours. Nous avons choisi de repérer les formes prises par quatre catégories de l'idéologique (Althusser, 1970) dans le discours. D'autres, avant nous, avaient fait appel au repérage de catégories identiques dans le discours de presse, en particulier pour analyser un corpus de romans-photos (Gretillat et al., 1981). Nous avons retenu les quatre catégories suivantes: 
- le sujet: une série d'abstractions catégorielles oppose le citoyensujet et les non-sujets. L'auteur et la victime sont concernés par cette catégorie. La police et la justice également, encore que leur activité puisse être rattachée à la catégorie "finalité" en tant qu'opérateurs.

- la scène: le lieu où se déroule l'action (la rue, une banque, un appartement privé, etc.); le moment de l'action (le jour, la nuit), les rapports entre l'endroit où se produit un acte délinquant et son environnement (la charge d'angoisse qui s'attache au lieu où le délinquant opère, etc.).

- la finalité: le message du journaliste est qu'il s'agit de remettre en son état initial la structure sociale. L'idéologie est là pour sacraliser certaines notions (Reboul, 1980). Ainsi en va-t-il à notre avis de la propriété privée. Reboul ajoute qu'il y a menace de violence pour ceux qui refusent la sacralisation ainsi opérée, d'où la justification de la prison, de la peine, pour ceux qui attentent à des espaces idéologiques protégés.

- l'action: les formes que prend le comportement de l'infracteur lorsqu'il contrevient à la loi; l'éventuelle réaction sociale qui s'ensuit pouvant aller jusqu'à la condamnation judiciaire. C"est au travers de l'action que peut se lire la fonction du récit.

\section{AUTEURS ET VICTIMES, LES ÉTERNELS SUJETS}

Althusser (1970) propose une démonstration aux termes de laquelle il n'y a d'idéologie que pour des "sujets concrets" et estime que l'idéologie interpelle les individus en sujets.

Or, lire un article de presse, c'est être confronté à un discours normatif condamnant des auteurs non conformes, s'adressant à des individus eux-mêmes sujets devenant à leur tour porteurs du discours entendu. Il s'agit forcément d'un discours créant des oppositions au sein du groupe social: auteurs/victimes, mais aussi auteurs/victimes potentielles que représente tout lecteur. Sous sa forme symbolique, le sujet social, atomisation des sujets, est un groupe implicite, le «dieu caché» (Guillaumin, 1972). Le groupe, ce sont les majoritaires opposés aux "mauvais sujets» qui postulent l'intervention de l'État par leur dangerosité. Au moment où s'opère l'opposition sujets/non sujets, peut se lire une liste d'exclusions à travers des signes désignant à la vindicte publique ceux-là mêmes qu'il s'agit d'éliminer, trahis par leur incapacité à demeurer sujets, dans l'expression bien souvent de la violence (Michaud, 1978), signes aggravés par le signalement de l'auteur, par les connotations attachées à la qualification de son acte, par l'emploi de stéréotypes, par la confusion de l'individu avec le signe. 


\section{LES SIGNES D'EXCLUSION DANS LA PRESSE}

Des signes à l'œuvre dans le corpus de presse désignent l'auteur de l'infraction. Certains sont des signes de base. Ce sont les signes les plus fréquemment à l'œuvre dans les récits analysés, mode d'identification du délinquant. Sur eux vont se greffer des signes dits "secondaires".

Signes de base:

- Jeune

- Étranger (le travailleur immigré essentiellement)

Signes secondaires:

- Toxicomane

- Récidiviste

- Masqué

- Armé

- Transgresseur de liens familiaux.

En quoi le signe se lira-t-il au second degré? Prenons pour exemple le cas du «toxicomane». Celui-ci renvoie à la représentation sociale qu'on a voulu en faire: un individu au regard fixe, aux cheveux longs, sale, mal habillé, jeune généralement. Parler de toxicomane équivaut à provoquer un déclic chez le lecteur, à reproduire un certain nombre d'images négatives. Le "récidiviste» relève du même mécanisme. Son apparence ne dit pas obligatoirement sa qualité, mais prononcer le qualificatif de "récidiviste" amène là encore à l'adoption d'un réflexe de méfiance, de vigilance, comme à l'égard de n'importe quel exclu.

Les signes énumérés, parfois cumulés pour un même auteur, se rencontrent dans les récits dont l'objet est de rapporter des faits divers relevant de la commission de soustractions de biens sans violence, vols essentiellement et actes de brigandage. S'agissant de délinquance d'affaires, la règle d'appartenance s'inverse et ce n'est qu'exceptionnellement que le délinquant d'affaires apparaît comme relevant des signes d'exclusion.

\section{A) CONNOTATIONS ET SIGNES D'EXCLUSION}

La connotation est couramment employée par tout langage idéologique. Parmi plusieurs termes possibles, un auteur va prendre un terme précis et non un autre. Bien entendu, le sort de la connotation est indissociable de la notion de norme. Admettre qu'il y ait un sens courant, c'est aussi poser pour acquis qu'existe pour un objet une dénomination normale, et pour un terme un sens courant, lequel se détermine par rapport au contexte. Pour un même objet, toute modification du contexte 
sera signifiée par l'emploi de connotations, valeurs surajoutées (KerbratOrecchionni, 1977; Barthes, 1970; Gary-Prieur, 1971).

La connotation n'existe que par rapport à la dénotation, sorte de présence d'un sens second, et une idéologie diffuse accentue l'effet de lexicalisation des appellations, banalise les figures, tout en utilisant aussi l'effet de tension entre le contexte et la figure. De plus, la valeur (connotative) attachée au signifiant linguistique et la valeur (dénotative) attachée au référent ont tendance à s'influencer mutuellement, c'est-àdire qu'un terme connoté "vulgaire" a tendance par un effet de contagion, à vulgariser le dénoté auquel il renvoie; le choix d'un terme de niveau "élevé" ou "bas" est un indice indirect du caractère prestigieux ou méprisable que le lecteur doit prêter au dénoté.

Connotations, vols et actes de brigandage dans les récits des deux quotidiens:

On prendra l'exemple du jeune:

Dans les faits divers de Nice-Matin:

Le signe "jeune" est associé à "toxicomane" dans tous les cas où est retenue une infraction commise par un toxicomane, tant dans Nice-Matin que dans la Suisse. De façon moins systématique, mais fréquemment, «jeune» est associé à "étranger".

Image d'une nature malfaisante:

"Ces jeunes loups du milieu marseillais».

Image inquiétante:

"Jeunes" est associé à "masqués"; n'ayant pas le contrôle d'eux-mêmes (surexcités).

Image malfaisante:

"Ces jeunes voyous assassins, appartenant à une nouvelle race de criminels", "malfrats", "diaboliques", "appartenant à une race de voyous».

Dans la Suisse, "jeune» n'est pas dominant, mais est avant tout associé à d'autres signes.

"Jeune» apparaît essentiellement lié à des infractions de brigandages, plus nombreuses dans Nice-Matin que dans la Suisse.

\section{Connotation et délinquance d'affaires}

Dans les deux journaux, les agissements des délinquants d'affaires s'organisent en fonction de la "profession» de l'auteur, ce qui donne une image plutôt rassurante. On décrit un individu qui a une couverture 
sociale non négligeable. À propos de la profession, le journaliste se permettra pourtant une certaine dérision; ou encore l'auteur sera montré comme irresponsable.

Mais plus la position sociale de l'auteur est importante, moins il sera dénigré.

Des motivations exceptionnelles ne peuvent que rendre le lecteur indulgent (l'employée de banque, jolie et amoureuse, détourne des fonds pour son amant).

À l'endroit des délinquants d'affaires, on rappellera le propos toujours actuel de Frégier, pour qui: «Le vicieux riche ou aisé qui dissipe son superflu et même une partie de son capital en plaisirs condamnables inspire le dégoût, mais non la crainte" (Frégier, 1840).

La délinquance d’affaires ainsi envisagée, apparaît moins comme un dysfonctionnement que comme une composante du système économique dans une société de classes (Kellens et Lascoumes, 1977).

Ces observations s'inscrivent bien dans un processus idéologique constamment à l'œuvre dans la presse. On est en face d'individus dont le comportement est a priori conforme à la rationalité dominante (Rigaux, 1974).

\section{B) SIGNES D'EXCLUSION ET STÉRÉOTYPES}

D'une manière générale, on peut dire que la propagande aboutit à la création de stéréotypes, et les besoins d'une réaction de masse qui caractérisent en premier lieu la propagande sont parfaitement satisfaits par les attitudes stéréotypiques. Les stéréotypes, dans l'usage qu'en font les médias, apparaissent comme des spécifications d'actualités (tel individu symbolisant le crime plutôt que tel autre) et c'est à l'aide de clichés nouvellement formés qu'on fera le plus facilement appel aux attitudes communes préexistantes. Cela peut aussi bien concerner des quotidiens couvrant une ville, voire une région entière, et sans doute plus par conséquent Nice-Matin (étant donné sa situation de monopole), que la Suisse. Merill et Lowenstein (1978) estiment que les masse-médias, par les nouvelles propagées, simplifient la réalité des événements, avec constance. Ils présentent les événements et les hommes, comme ayant une seule dimension, de manière statique.

Le stéréotype est une opinion toute faite, un cliché; c'est aussi "l'idée" que l'on se fait de... "l'image" qui surgit spontanément lorsqu'il $\checkmark$ agit de...: c'est la représentation d'un objet (choses, gens, idées), plus ou moins détachée de sa réalité objective, partagée par les membres d un 
groupe social avec une certaine stabilité (Bardin, 1977). Il correspond à une mesure d'économie dans la perception de la réalité, puisqu'une composition sémantique toute prête, généralement très concrète et imagée, organisée de quelques éléments symboliques simples, vient immédiatement remplacer ou orienter l'information objective ou la perception réelle. Structure cognitive acquise et non innée (soumise à l'influence du milieu culturel, des communications de masse), le stéréotype plonge ses racines dans l'affectif et l'émotionnel car il est lié au préjugé qu'il rationalise, justifie ou engendre. Le stéréotype est apparu en 1922 dans un ouvrage de Lippmann s'appliquant aux opinions et aux représentations sociales et désignant les «images dans notre tête" s'intercalant entre la réalité et la perception que nous en avons.

Et l'on sait que le recours aux stéréotypes a déjà été relevé comme caractérisant le fait divers en général (Auclair, 1982).

Le stéréotype est fréquemment employé dans Nice-Matin, il est beaucoup moins à l'œuvre dans la Suisse. Il vient renforcer les signes d'exclusion, particulièrement l'étranger, le jeune, le toxicomane.

\section{Stéréotypes pour vols et actes de brigandages}

Deux jeunes malfaiteurs armés... «ils avaient d'ailleurs masqué leur visage d'une cagoule». Tous les malfaiteurs sont-ils jeunes? On peut se le demander ici, alors que l'on nous présente des auteurs peu identifiables puisqu'ils ont recouvert leur visage. D'autant que dans le récit du journaliste, rien ne permet de penser qu'il s'agit d'individus d'un âge définissable. On pensera donc plutôt à une interprétation correspondant aux représentations de la délinquance chez le journaliste, donc à une reconstruction.

Les drogués prennent toujours pour cible les personnes âgées: «on ne peut donc que conseiller aux malades sur le point d'être hospitalisés de s'entourer d'un maximum de précautions... au risque d'être la cible des vauriens de la trempe de l'ambulancier sans scrupules».

D'une façon générale, les étrangers sont peu discrets dans leur tenue: le deuxième (agresseur) était petit, de race noire... "lui aussi, avait un tee-shirt de couleur bleue. Quant au troisième, plus discret dans sa tenue..."

\section{Stéréotypes et délinquance d'affaires}

L'emploi du stéréotype n'aggrave pas ici la représentation de l'auteur dans un sens de dangerosité et d'insécurité; il fait au contraire 
diversion dans Nice-Matin: l'escroc est présenté au bras d'une ravissante blonde ramenée dans ses bagages au moment où il s'apprête à s'enfuir (stéréotype de l'escroc, entouré de femmes). Ou alors la femme, ellemême escroc, est incapable de résister à l'amour (sous-entendu: la femme est faible); on on réfère donc pour l'ensemble à un fond stéréotypé déjà connu.

Nous renvoyons aux observations de Doise et Deschamp (1978) à propos du sociocentrisme (Piaget et Weil, 1951) qui serait à la base d'un stéréotype universel, que Lévine et Campbell ont décrit (le "nous" opposé au «ils»):

- nous sommes honnêtes et dignes de confiance entre nous, mais nous ne sommes pas dupes des combines des étrangers;

- nous sommes courageux et allons de l'avant; nous défendons nos droits et nos propriétés; nous ne nous laissons pas marcher dessus;

- nous sommes moraux et propres, etc.

Ce qui a trait au stéréotype déborde donc largement le groupe étranger et s'applique à tout ce qui est différent du groupe dominant.

\section{STRATÉGIES D'ARGUMENTATION ET IMAGES}

Il y a différentes façons de rapporter un événement et on doit pouvoir tirer des conséquences de l'articulation du récit de presse en ne perdant pas de vue que des images précises naissent dans l'esprit de celui qui lit un article des éléments mis en avant par la narration elle-même.

Il s'agit de montrer comment est mise en scène la délinquance au travers des divers éléments d'un discours, en essayant de voir comment, au-delà de la manière dont s'agencent des faits précis, se profile l'idéologie du journaliste. Car, ce que restitue le journaliste dans un article, ça n'est pas le réel, mais sa propre reconstruction du réel puisque tout discours idéologique fonctionne à la transformation (De La Pradelle, 1978).

Pour étudier les stratégies d'argumentation et la mise en scène, nous avons retenu huit récits. Nous nous y sommes attachés à l'étude de l'axe syntagmatique en examinant de quelle façon se mettaient en place les modes d'argumentation du journaliste lorsque ceux-ci apparaissaient. Deux opérations principales: la première consistant à dégager les niveaux discursifs d'un texte en respectant leur ordre d'apparition (Vignaux, 1972); la deuxième nécessitant de faire apparaître les adhésions ou les négations, voire les rejets formulés par le journaliste, mettant à nu au 
travers d'un agencement du récit sa propre représentation du délinquant (Maingeneau, 1979).

Deux aspects apparaissent centraux dans les huit récits participant de la mise en scène: individualisation et abstraction. Individualisation signifie tendance à expliquer l'action par des caractéristiques propres à l'auteur qui l'éloignent d'autres catégories sociales telles les jeunes, les immigrés, la drogue, etc. L'individualisation poussée à son extrême va faire du cas traité une situation unique, non susceptible d'atteindre le lecteur. Elle s'oppose en principe à l'abstraction, qui, au contraire, fait de l'auteur une sorte de miroir d'une myriade d'individus, tous analogues, voleurs potentiels risquant de s'attaquer au lecteur. Il arrive qu'un dosage d'individualisation (récit axé sur l'histoire personnelle de l'auteur) et d'abstraction (récit faisant appel également au milieu dans lequel évolue l'auteur) soit à l'œuvre dans le récit.

\section{CONTRADICTIONS DU JOURNALISTE}

On peut se demander si une contradiction ne naît pas d'intentions peu compatibles du journaliste, surtout dans Nice-Matin, à savoir, conserver un certain consensus au sein des lecteurs et exploiter un matériau à sensation. Le maintien d'un consensus implique la description d'un monde atypique, l'évitement de réalités alternatives potentielles offertes par le délinquant, toutes choses que risque, bien au contraire, de souligner le journaliste en mettant l'accent sur l'activité du délinquant. Prenons l'exemple de celui qui commet un hold-up: il va se retrouver en possession d'une somme d'argent considérable lui permettant de s'offrir des plaisirs réservés à d'autres catégories sociales (participation au monde des classes dirigeantes et aisées). Cet aspect du vol, tendant à en faire un moyen de s'enrichir à bon compte, est en totale contradiction avec les fondements du contrôle social: maintien des rôles, délimitation très stricte de la liberté.

Autre contradiction: le journaliste se doit de faire l'apologie de l'homme en tant qu'agent libre et, simultanément, il se doit de pousser le lecteur à l'indignation contre l'atteinte à la propriété d'autrui relevant sous un certain angle de la liberté de comportement. Pour résoudre la contradiction, le journaliste va étiqueter le délinquant au moyen du signe d'exclusion. Le lecteur (majoritaire), dit Young (1979) se tient sur une plate-forme néo-keynésienne, à une seule dimension et anhistorique: il a décidé de travailler et d'être un bon consommateur. La profession du délinquant et le détoumement opéré sur le cours de l'activité professionnelle, suffisent à faire le partage entre délinquance économique et vol, comme si dans le premier cas la soustraction était quasiment intégrée à 
l'activité professionnelle, ou dans des cas moins extrêmes, atténuait la responsabilité.

Anhistoricité encore, en ce qu'en naturalisant les notions de propriété, de liberté, d'égalité, on évacue toute l'exploitation et l'inégalité (Thévenin, 1974), pour en arriver à un discours universaliste (Reboul, 1980). On passe ici sous silence les racines de la propriété privée et les structures sociales qui les fondent (Pasukanis, 1970).

\section{VIE ET ARGENT}

L'ensemble des récits privilégie la vie par rapport à l'argent dans la façon de fustiger la délinquance, la vie étant un bien plus important que les biens matériels, au sein d'une société où, paradoxalement, l'argent est souvent le moteur de l'action. Peut-être, pour en juger, faut-il reprendre le propos d'Althusser (1970) pour lequel l'idéologie donne une image inversée des rapports sociaux. Car, en présentant au lecteur sa vie comme son bien le plus précieux, le journaliste ne donne-t-il pas, du même coup, l'illusion d'une égalité entre êtres humains dans un monde où l'inégalité se manifeste justement essentiellement au niveau de l'argent? On adhérera d'autant plus à cette idée qu'en d'autres cas, et lorsqu'il ne s'agit plus d'utiliser cette fois l'intégrité physique comme facteur d'égalisation entre les individus, l'importance de la vie humaine est négligée: on pense aux accidents du travail, aux accidents de la circulation faisant chaque année en France et ailleurs des milliers de morts, thème que le journaliste, se garde de traiter dans les mêmes termes que les atteintes aux biens violentes, les accidents de la circulation risquant de mettre en cause le culte de l'automobile (Chesnais, 1981). La menace de la vie humaine n'est soulignée que lorsque'elle semble utile à la cohésion sociale: en attentant à une parcelle de peau et de vie d'un sujet, l'agresseur attente à la vie même du sujet (l'ordre social). Pourtant, dit, Chesnais: «en sortant dans la rue, on risque davantage d'être écrasé par une voiture qu'attaqué par un malfrat... La route est le seul lieu où le crime soit permis en toute impunité".

\section{3. ÉGALITÉ}

La mise en scène du crime s'inscrit dans une structure sociale qui privilégie l'égalité nécessaire à la notion du sujet. Pour être admissible, la notion de propriété privée sous-tendant le droit pénal se doit d'appartenir à une société égalitaire.

L'égalité, reflet inversé des rapports réels (Labica, 1964) gouverne l'apparence qui les donne à voir, et ses catégories, telles "les notions 
juridiques de personne et de liberté». Car le droit est constamment sous-jacent dans l'édifice idéologique dont la mise en scène observée là n'est qu'un des aspects. C'est bien parce qu'il y a proclamation et mise sous surveillance du droit de la propriété privée qu'il peut y avoir description des diverses formes que prennent les atteintes aux biens.

Le droit, que l'on retrouve à l'œuvre dans les comptes rendus de procès, est chargé d'assurer ici l'efficacité de la circulation des biens (Edelmann, 1973), de sanctionner tout «accident de parcours». Il apparaît à ce point de l'analyse que le droit pénal met en cause quatre notions essentielles qui sont: le sujet, la propriété, l'égalité et la liberté, et que la mise en scène du crime s'inscrit dans un cadre borné par ces notions-là.

\section{MISE EN SCÈNE DU CPIME ET DE L'ÉCONOMIE}

Celles-ci s'alignent l'une sur l'autre: la mise en scène du crime met en pleine lumière une atteinte à la propriété privée, tandis que la mise en scène de l'économie tient dans l'ombre cette obscure entreprise où l'on produit de la plus-value et que seule paraît sur l'écran l'image d'un marché où la propriété s'échange librement, le droit étant là pour garantir cet échange, la liberté des contrats et l'égalité des prestations fournies.

La sphère de circulation est un «éden" où croissent "naturellement" les droits de l'homme, où les catégories de liberté et d'égalité sont, à ce titre, des déterminants de la valeur d'échange (Dujardin, 1979). Ces notions sont fondamentales dans la mise en place d'une idéologie renfermant l'obligation de respecter la propriété privée: comme par définition nous sommes égaux, celui dont l'avoir dépasse celui des autres l'a forcément gagné par des voies légitimes. Celui qui remet en cause indirectement ce postulat, en laissant entendre dans un acte de transgression que l'autre a acquis injustement un bien puisqu'il veut se l'approprier, doit être revêtu d'un signe d'exclusion.

Smaus (1982) note que la propriété privée revêt une importance encore plus ambivalente que la protection de la vie. La propriété, aussi infime soit-elle, est douée d'une fonction anthropologique qui lui permet de produire un consensus. La signification subjective qu'a la propriété pour chacun explique pourquoi ce sont précisément les empiètements mineurs qui sont si fortement marqués symboliquement par les organes de contrôle. Car derrière l'intelligibilité universelle de la propriété, on peut en effet efficacement cacher sa répartition inégalitaire. 


\section{ANCRAGE DES RÉCITS DANS L'ABSTRACTION JURIDIQUE}

On notera aussi que l'on assiste à l'ancrage de la mise en scène du crime dans l'abstraction juridique. Alors que pour Miaille (1976) les notions de droit sont présentées et traitées en dehors d'un contexte social précis, on assiste ici au traitement du fait sans énonciation des règles idéologiques qui conduisent le journaliste à en faire une certaine utilisation. Il n'empêche que l'abstraction à l'œuvre avec les signes d'exclusion analysés plus haut est celle-là même qui régit le moule juridique, même s'il s'agit de lui faire remplir un rôle différent. L'abstraction relevée à propos de la mise en scène de la délinquance a pour tâche d'étiqueter des individus; celle à l'œuvre dans la règle de droit a pour objectif de signifier l'application à tous de textes qui en réalité gouvernent alternativement divers groupes sociaux: si le droit pénal, dans une logique que respecte la mise en scène du crime, a pour effet de stigmatiser les couches de la population se situant au bas de l'échelle sociale, le droit des sociétés ou la liberté de choisir son régime matrimonial n'intéressent guère que les couches moyennes et aisées (De la Pradelle, 1978). C'est pourtant toujours la même abstraction qui est à l'œuvre. La mise en scène du crime, son côté spectaculaire, font oublier l'existence d'inégalités sociales qui peuvent être motrices dans la commission d'actes constitutifs d'atteintes aux biens. La peur des individus-lecteurs paralyse toute interrogation sur la fortune considérable détenue par certains, dérobée pour partie sous leurs yeux; la peur et également la projection (ça peut m'arriver aussi), à propos de vols sans commune mesure. On notera que, de la même façon sur un plan purement économique, le fétichisme fait oublier que la production et la circulation des objets appelés marchandises cachent en réalité des rapports sociaux entre les individus. L'économie, comme le souligne Miaille, serait le lieu de la production et de la distribution des richesses. Celles-ci seraient extraites de la nature, pour faire l'objet d'échanges, mais jamais n'apparaîtraient les rapports entre les hommes qui permettent l'organisation de cette production et cette circulation.

La mise en scène du crime est soumise de façon sous-jacente à des règles universelles qui le rendent prévisible, celles d'un monde juste et manichéen où les bons, protégés par des institutions répressives aidées par la fatalité, l'emportent sur les méchants. On montre au lecteur un monde où les méchants sont marqués d'indices qui les rendent dangereux.

On sera tenté de faire un parallèle entre les mille visages de la mise en scène du crime et ceux relevant de la mise en évidence du politique dont parle Balandier (1980), la société ne «tenant" pas seulement par le jeu de la coercition et des rapports de force légitimés, mais aussi par 
l'ensemble des transfigurations dont elle est à la fois l'objet et le réalisateur, transfigurations dont participe l'idéologie à l'œuvre dans la presse.

La mise en scène du crime témoigne des codes culturels contemporains, fustigeant ce qui relève de l'interdit par la définition du sujet (auteur-victime), sa mise en scène et son action. Sont stigmatisées des relations considérées comme pathologiques, correspondant à des attitudes précises, non pas dans un sens psychosociologique, mais dans la compréhension de comportements très concrets, de modes d'expression (gestuels, verbaux, etc.) des auteurs.

Ainsi se présente donc le contenu d'une presse régionale, reste à savoir de quelle façon cette presse influe sur ses lecteurs.

\section{DANS QUELLE MESURE LA PRESSE FAÇONNE-T-ELLE LES DISCOURS PRIVÉS?}

On a longtemps parlé de "l'effet miroir" des médias (Klapper, 1976), puis cette thèse a été relativisée (Noëlle Neumann, 1977). Ici un certain nombre de faits montrent que la presse régionale imprègne moins fortement qu'on aurait pu le croire les représentations de ses lecteurs.

Il est vrai que la presse n'est pas seule à influer sur les représentations de ses lecteurs. Ceux-ci s'abreuvent à d'autres sources qui sont principalement:

- des réseaux privés. Nous appelons ainsi des réseaux dans lesquels chaque individu est intégré et à l'intérieur desquels il reçoit toutes sortes d'informations concernant - entre autre chose - la commission de crimes. Comme le note Dulong (1981), il reste dans la représentation de l'expérience narrée par autrui une part fantasmatique qui amène chacun à être davantage que victime personnellement, ou en danger de l'être, s'appropriant le vécu d'autrui.

- des rumeurs, souvent colportées par les réseaux. Car, malgré les progrès technologiques, le mode de communication social le plus permanent est toujours celui du ouï-dire, de la bouche bavarde qui se penche sur l'oreille curieuse (Flem, 1981). Les rumeurs concernant les comportements déviants des étrangers, voire des jeunes, ne manquent pas.

De multiples travaux ont été menés en Europe sur l'impact des médias en matière de représentation du crime. Et notamment par Schneider (1978) pour le Conseil de l'Europe. Également par Van Dijk aux Pays-Bas. Aux termes de sa recherche, Van Dijk (1980) montre que les journaux aussi bien que les télévisions abreuvent la population d'images stéréotypées sur le crime et les criminels. Les messages des médias ont 
une très grande influence sur les femmes, les personnes âgées et les gens à faible niveau d'éducation. D'après Smaus (1983) recensant les travaux allemands récents relatifs aux effets des masse-médias sur le crime, bien que de multiples ouvrages se rapportant à ce thème puissent être dénombrés, on n'y trouve cependant pas de travaux comparables à ceux un peu plus anciens de Vince, Klapper, Halloran, Brown et Chaney. La plupart des approches ne vont pas au-delà des considérations de nature spéculative ou morale et elles n'ont suscité pratiquement aucun intérêt dans la littérature sociologique récente.

En France, Mouillaud (1981) estime que le discours de presse ordonne un champ propre, un champ de lecteurs. Lascoumes, MoreauCapdevielle et Vignaux (1978) appréhendent les lecteurs comme des acteurs. Pour Faugeron (1978), le discours de presse joue plutôt un rôle de révélateur et de renforcement que de création.

Nous constatons nous aussi que les messages de presse foumissent des schémas explicatifs qui sont ensuite réutilisés pour étayer des représentations déjà existantes (Faugeron, 1978). Ainsi va-t-on se servir de l'analyse du journal lui-même pour toute une théorie sur le contenu des journaux lorsque l'on est un intellectuel ou que l'on appartient aux professions libérales quel que soit son groupe de rattachement, en ayant une position critique sur la presse en général. «La manière dont sont écrits certains articles, c'est énorme", nous dit-on. Du même coup, la délinquance voit son augmentation liée à l'existence d'une certaine presse, puisque, dit le même interviewé, "je suis sûr que ça doit encourager beaucoup de délinquants de lire les journaux..." (sociologue genevois de 36 ans).

Les journaux régionaux servent bel et bien dans la mise à distance de la peur du crime lorsqu'on élabore une construction au sein de laquelle les quotidiens sont considérés comme cherchant à détourner l'attention des gens de leurs difficultés: on essaie de polariser l'attention des gens sur des problèmes, sur des faits divers, sur des choses comme ça, ça peut-être la délinquance...". La peur produite est artificielle, sans fondement réel et le moyen trouvé est simple: "les premiers titres sur la première page c'est toujours quand même des faits, des faits divers ou des hold-up ou des choses comme ça...»

La presse régionale s'intègre parfaitement aussi dans un discours froid, rationnel et bien rôdé sur la délinquance; ainsi va-t-on se servir des journaux locaux pour convaincre l'interlocuteur de ce que le petit vol est en augmentation: "Je viens de lire ce matin dans le journal la Suisse un article sur un super-marché qui s'est ouvert, je ne sais plus si c'est à Nyon 
ou à Neuf-Châtel, je ne me rappelle plus, mais enfin... on signalait je crois qu'il y avait 28 ou 30 vols, on avait arrêté 28 ou 39 voleurs l'année dernière" (le même sociologue).

On prend appui sur la presse pour étayer la thèse selon laquelle il n'y a que la violence qui intéresse autour de soi, et d'ailleurs dit-on, les journaux sont remplis de cette violence, il n'y a que cela qui intéresse les gens. C'est une variante du thème vu précédemment dans lequel, on utilise la délinquance pour détourner l'attention des gens de leurs difficultés de vie.

La presse régionale est là encore pour étayer sa propre croyance à l'augmentation du crime. Ainsi chez cette interviewée qui déclare à propos de la lecture du journal: "Non, c'est tout du fait divers, c'est le train-train quotidien, on a l'habitude qu'il y ait des cambriolages, des attaques, enfin, maintenant je pense, bon il y a 20 ans ou 30 ans en arrière, c'était différent, mais maintenant ça s'est banalisé, on sait très bien qu'il y a des gens qui vont vous voler une fois, vous avez toutes les chances d'être volé une fois dans votre vie, et puis voilà" (commerçante âgée de 30 ans à Nice).

La presse renforce cette idée que l'on a d'un manque de discipline chez les jeunes, de leur liberté trop prononcée, ou encore de ce que les gamins mériteraient d être beaucoup plus poursuivis, jugés de façon plus sévère.

Enfin, des individus victimes d'une infraction, citent la presse locale à l'occasion de leur propre mésaventure, dans le sens qui les arrange. Une interviewée ouvrière à Genève déduit du silence du journal local sur sa mésaventure, remarquant par ailleurs le signalement de vingt cinq vols commis ce jour là, que ceux-ci étaient plus graves que le sien (qui l'a pourtant grandement traumatisé), ceci étant un moyen de réduire la peur (sous-entendu: "j'ai eu peur, mais d'autres ont encore plus que moi des raisons d'avoir peur"). Ce qu'on lit est alors toujours pire que ce que l'on subit. Manseau et Normandeau (1981) foumissent l'exemple d'une victime qui spécifie que la version des journaux de l'événement qu'elle relate est fausse, ce qui ne l'empêche pas de croire ce que les journaux racontent au sujet d'autres vols. Mais la même démarche peut aussi alimenter la peur du vol: les journaux, même si leurs colonnes regorgent de faits divers, n'en publient qu'une infime partie: «parce que les journaux, moi je vous dis qu'on en met très peu hein, il y en a quand même toujours un petit échantillonnage, mais surtout par le commerce, le travail, alors là je vous dis c'est tout le temps, tout le temps (employée niçoise de 57 ans). 
Ceci va bien dans le sens de ce que prétendait déjà Verneuil (1981) pour qui la population ne reçoit pas passivement les messages qui lui sont régulièrement délivrés. Les discours sur la criminalité et le système pénal rencontrent des structurations dont on s'aperçoit qu'elles sont de véritables actrices cachées dans la formation des représentations. Zauberman (1982) a sans doute raison, s'appuyant sur les travaux de Hallet al. (1978) de parler d'un continuel va et vient entre médias et lecteurs.

\section{EN CONCLUSION}

Le lecteur du journal - ici régional - consomme tranquillement le crime, disposant grâce à son quotidien habituel d'un volet de références pour asseoir ses représentations. Les récits sont semblables à de courtes séquences de films, décors et personnages en miroir dont il ne retient que quelques archétypes: le jeune, le toxicomane, l'étranger... La presse permet à l'individu de renforcer des opinions préexistant à l'opération de lecture et il y a manipulation du fait médiatique par ses lecteurs.

L'article de journal donne à voir signes et images, formes qui se figent à la lecture et dont on ne sait plus bien si elles tiennent de la réalité ou de la fiction. Cela est sans importance, puisque dans tout spectacle la limite entre le réel et l'imaginaire est censée se déplacer à l'infini.

\section{BIBLIOGRAPHIE}

ACKERMANN, W., "Séminaire", l'Imaginaire de l'insécurité, Paris, 1982.

ALTHUSSER, L., la Pensée, juin 1970, p. 1 et s.

ARNAUD, A.J., l'Analyse structurale du code civil, Paris, L.G.D.J., 1973.

AUCLAIR, G., le Mana quotidien, Paris, Anthropos, 2e éd., 1982, pp. 141.

BALANDIER, G., le Pouvoir sur scène, Paris, Ballaud, 1980.

BARATTA, A., "Remarques sur la fonction idéologique du pénitencier dans la reproduction de l'inégalité sociale*, Déviance et Société, 1982, vol. V, p. 121 et s.

BARDIN, L., l'Analyse de contenu, Paris, P.U.F., 1977.

BARTHES, R., S/R, Paris, Le Seuil, 1970.

BERNSTEIN, Langage et classe sociale, Paris, Minuit, 1976.

BOURDIEU, P., "La jeunesse n'est qu'un mot”, dans Questions de sociologie, Paris, Minuit, 1980, p. 151.

CHESNAIS, J.C., Histoire de la violence, Paris, Laffont, 1981, pp. 277 et s.

DESCHAMPS, J.C., l' Atribution de la catégorie sociale, Beme, P. Lang, 1977, pp. 46 et ss. 
DOISE, W., J.C. DESCHAMPS, G. MUGNY, Psychologie sociale expérimentale, Paris, Colin, 1978.

DUJARDIN, P., 1946, le Droit mis en scène, Grenoble, P.U.G., 1979, pp. 25 et s.

DULONG, R., l'Auto-défense, Paris, Librairie des Méridiens, 1983.

DULONG, R., les Deux pôles de l'imaginaire et de l'insécurité, auto-défense et hyperprotection, Séminaire du Centre d'études sociologiques sur l'imaginaire de l'insécurité, Paris, 1981.

EDELMAN, B., le Droit saisi par la photographie, Paris, Maspero, 1973, pp. 92 et s.

FAUGERON, C., «Du simple au complexe: les représentations sociales de la justice pénale", Déviance et Société, 1978, 2, 4, 411-432.

FLEM, "Bouche bavarde et oreille curieuse», dans le Genre humain, $\mathrm{n}^{\circ} 5,1981$, p. 13.

FRÉGIER, M.A., les Classes dangereuses de la population dans les grandes villes, Bruxelles, 1840, cité par Van de Kerchove, M., Culpabilité et dangerosité, dans Ch. DeBuyst (édit.), Dangerosité et justice pénale, Genève, Médecine et Hygiène, 1981.

GABEL, J., la Fausse conscience, Paris, Minuit, 1962.

GALTUNG, J., M. RUGE, "Structuring and Selecting News", dans S. Cohen, J. Young (édit.), The Manufacture of News Social Problems, Deviance and the Mass Media, London, Constable, 1974, pp. 62-73.

GARY-PRIEUR, M.N., "La notion de connotation», Littérature, décembre 1971, p. 96.

GRETILLAT, F., J.P. KELLER, J. KELLERHALS, L. VONECHE, «Une relation sans échange", Revue suisse de sociologie, 1981, vol. 7, 1 et 5.

GUILLAUMIN, C., l'ldéologie raciste, La Haye, Mouton, 1972.

HALL, S., C. CRITCHER, T. JEFFERSON, J. CLARKE, B. ROBERT, Policing the Crisis, London, Mac Millan press, 1978.

KELLENS, G., P. LASCOUMES, «Moralisme, juridisme et sacrilège: la criminalité des affaires", Déviance et Société, 1977, I, p. 129.

KERBRAT-ORECCHIONNI, C. , la Connotation, Lyon, Presses universitaires de Lyon, 1977, p. 219.

KLAPPER, J.J., The Effects of Mass Communication, Glencoe, The Free Press, 1960.

LABICA, G., Dialectiques, 1964, n 6, p. 60 .

LASCOUMES, P. et G. MOREAU-CAPDEVIELLE, «Presse et justice pénale, un cas de diffusion idéologique", Revue française de sciences politiques, 1976, XXVI, 1, 41-69.

LASCOUMES, P., G. MOREAU-CAPDEVIELLE, G. VIGNAUX, "Il y a parmi nous des monstres", Communications, 1978, XXVIII, 127-163.

LUDWIG-BOLTZMANN - Institut für Kriminalsoziologie, "Materialien zur Kriminal und Sicherheitsberichter-stattung", Kriminalsoziologische Bibliographie, 1976, IV, 11-13, pp. 52-67.

MAINGENEAU, D., Initiation aux méthodes de l'analyse du discours, Paris, Hachette, 1979.

MANSEAU, H., A. NORMANDEAU, "Les victimes de vol à main armée", Revue de droit pénal et de criminologie, juin 1981, p. 547. 
MEMMI, A., le Racisme, Paris, Gallimard, 1982.

MERRIL, J.C., R. LOWENSTEIN, Media, Message and Men, New York, Longman, 1978.

MIAILLE, M., Une introduction critique au droit, Paris, Maspero, 1976, pp. 119 et s.

MICHELAT, G. et M. SIMON, Classe, religion et comportement politique, Paris, Presse de la Fondation nationale des sciences politiques, 1977.

MOUILLAUD, M., "Métro, la peur; violence entre le "poids des mots et le choc des photos", Lumière et vie, 1981, XXX, 152, 56-72.

NOËLLE-NEUMANN, E., Offentlichkeit als Bedrohung Beiträge zur empirischen Kommunitiosforschung, Freiburg i. B., Albert, 1977.

PASUKANIS, E.B., la Théorie générale du droit et le marxisme, Paris, E.D.I., 1970, p. 57.

PIAGET, J., J. WEILL, «Le développement chez l'enfant de l'idée de patrie et des relations avec l'étranger», Bulletin International des sciences sociales, Paris, U.N.E.S.C.O., [951, pp. 605-62].

De La PRADELLE, G., l' Homme juridique, Paris, Maspero, 1978, pp. 28 et s.

QUERÉ, L., Des miroirs équivoques, Paris, Aubier, 1982.

REBOUL, O., Langage et idéologie, Paris, P.U.F., 1980.

RIGAUX, F., Introduction à La science du droit, Bruxelles, Éditions Vie ouvrière, 1974.

ROBERT, Ph., la Question pénale, Genève, Droz, 1984.

SCHNEIDER, M.H.J., L'influence des moyens de communication de masse sur l'opinion publique en matière de crime et de justice criminelle, Rapport présenté à la treizième conférence de recherche criminologique, Strasbourg, 1978.

SMAUS, G., "L'image du droit pénal et la reproduction idéologique des classes sociales», Déviance et Société, 1982, vol. VI, p. 355.

SMAUS, G., "Mass media et criminalité: état de la recherche en Allemagne et en Autriche", Déviance et Société, 1983, vol. VIl, ${ }^{\circ} 3$, p. 249 et $\mathrm{s}$.

STEIN-HILLBERS, M., Kriminalität im Fernsehen, Stuttgart, Enke Verlag, 1977, pp. 62 et ss.

THÉVENIN, N.E., «Idéologie juridique», la Pensée, 1974, p. 81.

VAN DIJK, J.J.M., «L'influence des médias sur l'opinion publique relative à la criminalité: un phénomène exceptionnel?", Déviance et Société, 1980, vol. IV, $\mathrm{n}^{\circ} 2$, p. 107 et $s$.

VERNEUIL, D., "Fonctions et processus du système pénal”, Déviance et Sociésé, 1981, vol. V, p. 223.

VIGNAUX, G., "Le discours argumenté écrit", Communications, 1972, $n^{\circ} 19$, pp. 101 et ss.

YOUNG, J., “Mass Media, Drugs and Deviance», dans Deviance and Social Control sous la direction de Rock P. et McIntosh, London, Tavistock, 1979.

ZAUBERMAN, R., "La peur du crime et la recherche", Année sociologique, 1982, p. 415. 